\title{
Scientists' attitudes toward a dialogue with the public: a study using "science cafes"
}

\author{
Eri Mizumachi, Kentaro Matsuda, Kei Kano," Masahiro Kawakami, Kazuto Kato
}

\begin{abstract}
Currently, science is developing rapidly and its influence on society is more significant than ever. This is all the more reason for today's scientists to interact with the general public. To design effective science communication activities, we must understand scientists' motivations and barriers to publicly communicating science. In this study, we interviewed 19 early-career scientists who had participated in science cafes in Japan. From these interviews, we identified five factors leading to their reluctance to participate in science cafes: 1) troublesome or timeconsuming; 2) pressure to be an appropriate science representative; 3) outside the scope of their work; 4) could not perceive any benefit; and 5) apprehension about dialogue with the public. Among these factors, apprehension about dialogue may be the clearest reflection of the scientists' underlying feelings about this form of communication and an indicator of more intrinsic barriers to engaging in science cafes.
\end{abstract}

\section{Introduction}

The rapid development of science and its ever-growing influence on society make it imperative that researchers recognize the social impact and meaning of their research, as well as actively engaging with the general public. ${ }^{1}$ Scientists and the public are expected to discuss not simply the science itself, but how science affects society. This trend is accelerating within several scientific disciplines, such as stem cell research, ${ }^{2}$ nanotechnology, ${ }^{3,4}$ neuroscience, ${ }^{5}$ genome science, ${ }^{6}$ and ecology. ${ }^{7}$ To achieve the objective of effective public dialogue, scientists and the broader scientific community have been increasingly engaging in a variety of science communication activities.

Several surveys about scientists' attitudes toward science communication have been conducted. ${ }^{1,8-14}$ Although about half of the scientists surveyed agreed that they had a duty to communicate their research and its implications to the public, ${ }^{9,14}$ most scientists also have complaints about the experience of science communication activities, such as the resulting time constraints, receiving little support from their peers, and offering little benefit. ${ }^{1}$ However, very few surveys have focused on the difficulties encountered by scientists with some experience in public science communication. To promote more effective and active science communication activities, it is essential to identify scientists' perceived barriers and motivations toward such activities.

In Japan, White Paper on Science and Technology 1993 showed that more young Japanese were moving away from science and technology, and it suggested that moves be made to promote a society where the public would be more aware of science and technology. ${ }^{15}$ In response to the white paper's suggestion, the First-Stage Basic Plan for Science and Technology in 1996, instigated by Cabinet council in Japan, indicated the future direction for promoting the public understanding of science (PUS) activities. ${ }^{16,17}$ For example, a national program facilitating interactions between researchers and formal education (Science Partnership Program) was introduced in 1996. The Second-Stage Basic Plan for Science and Technology in 2001 indicated ways to promote not only PUS, but also researchers' understanding of society. ${ }^{18}$ Against this background, one of the authors of present study, Kato since 2002 has held a public event called Genome Square, ${ }^{6}$ which is a unique opportunity to bring the Japanese public and genome researchers into face-to-face communication.

\footnotetext{
${ }^{*}$ These authors contributed equally to this work.
} 
In 2005, the Japanese government initiated the support for science communication by establishing training programs for science communicators at three universities (Hokkaido University, The University of Tokyo, and Waseda University) and funding public outreach activities. Moreover, the same year, two universities (Ochanomizu University and Tokyo Institute of Technology) initiated science communication programs, and Osaka University established the Center for the Study of Communication Design. Also in 2005, National Museum of Emerging Science and Innovation, Tokyo (Miraikan) started training programs for science communicators, and the National Museum of Nature and Science, Tokyo began preparing science communicator training programs. Therefore, the year 2005 is considered the first year of science communication in Japan. ${ }^{19}$ Since then, science communication has been embedded in government science and technology policy, and it has received great attention, especially within the scientific community. ${ }^{20}$ Since April 2011, scientists in Japan who receive over 30 million yen in public funds have been obliged to communicate science and its benefits with the public, and so science communication will assume an increasingly important role. ${ }^{21}$ The present study, which was carried out in 2009, was therefore conducted in a progressive era of science communication in Japan.

Science cafes, also known as cafes scientifiques, were launched in the UK and France around 1997 as a product of the contemporary science communication movement. ${ }^{20}$ They differ from PUS in that the aim is to discuss and question, in an open-minded manner, the consequences of scientific research and how science is being practiced ${ }^{22}$. Over the last decade, science cafes have expanded their presence in several countries. ${ }^{23,24}$ In Japan, the science cafe movement is said to have started in 2005, having been triggered by a column about introducing such cafes in White Paper on Science and Technology $2004 .{ }^{19,25}$ Since then, science cafes have become a popular and casual forms of public dialogue.$^{20,26,27}$ Now, over 100 science cafe organizers, including universities, research institutes, NPOs, local governments, bookshops, motivated individuals, and volunteer groups, organize science cafes on a regular or occasional basis in Japan. Science cafes have thus become appropriate venues where scientists can clarify their attitudes to the public.

In this general movement, the Japanese government has played an important role and promoted science communication activities; most developments were not initiated by the scientific community. In this context, it is necessary to analyze what attitudes scientists have adopted toward science communication. Based on an Internet-based questionnaire survey among Japanese life science scientists, Shineha et. al. suggested that the infrastructure and new communication tools and designs were important to promote science communication. ${ }^{12}$ A qualitative study was therefore needed to facilitate further analysis of scientists' fundamental attitudes to engaging in science communication.

\section{Objective}

Our objective was to identify scientists' attitudes, motivations, and barriers to participating in science communication activities in the form of science cafes.

\section{Methods}

\section{iCeMS Cafes}

A series of science cafes called the iCeMS Cafes was hosted by the Institute for Integrated Cell-Material Sciences (iCeMS) of Kyoto University. The iCeMS Cafes were organized by the iCeMS's Science Communication Group, which is made up of three of the present authors (Kano, Mizumachi, and Kato). Five iCeMS Cafe events were held between March 2008 and February 2009. Table 1 outlines the theme of each iCeMS Cafe, the research field of the guest speakers, and the number of scientists who participated. In an iCeMS Cafe event, an iCeMS principal investigator and early-career scientists from the investigator's laboratory held face-to-face conversations with the public while drinking tea or coffee in a relaxed, friendly atmosphere. The principal investigator selected the early-career scientists who participated in the iCeMS Cafe from their laboratories. A total of 27 early-career scientists attended the five iCeMS Cafes. 


\begin{tabular}{|c|c|c|c|c|c|}
\hline & Year & Date & Theme & Research Field & $\begin{array}{l}\text { Number of } \\
\text { Early-Career } \\
\text { Scientists }\end{array}$ \\
\hline No. 1 & 2008 & March 1st & " House for Molecules, Made to Order " & $\begin{array}{l}\text { Coordination } \\
\text { Chemistry }\end{array}$ & 5 \\
\hline No. 2 & 2008 & May 10th & $\begin{array}{l}\text { " See the Unseen - How molecules work } \\
\text { in your body?" }\end{array}$ & $\begin{array}{l}\text { Single-Molecule } \\
\text { Physiology }\end{array}$ & 4 \\
\hline No. 3 & 2008 & August 2nd & $\begin{array}{l}\text { "Transport - Discriminate Need and } \\
\text { Unneed " }\end{array}$ & Cellular Biochemistry & 7 \\
\hline No. 4 & 2008 & $\begin{array}{l}\text { December } \\
\text { 23rd }\end{array}$ & $\begin{array}{l}\text { "Chemical Tools for Exploration of } \\
\text { Biology" }\end{array}$ & Chemical Biology & 6 \\
\hline No. 5 & 2009 & $\begin{array}{l}\text { February } \\
21 \text { st }\end{array}$ & "Shapes in the Brain" & $\begin{array}{l}\text { Developmental } \\
\text { Neurobiology }\end{array}$ & 5 \\
\hline
\end{tabular}

Table 1. iCeMS Cafes held in 2008-2009.

At each iCeMS Cafe, 20 to 30 members of the general public took part; these participants were randomly selected from among applicants who had learned of the science cafe through Web sites, newspapers, or e-mail-alerts. At iCeMS Cafe, five to six discussion tables were prepared and one scientist and four to seven participants had a dialogue over each. In all five events, there was a total of 135 participants, comprising 76 males and 59 females (i.e., 56.3\% to 43.7\%), and the participants were diverse in age and profession (for example, high school teachers, painters, office workers, students, housewives, and retired persons).

Each iCeMS Cafe consisted of two parts. Initially, the principal investigator introduced his or her research theme for 15-20 minutes. We requested that these investigators briefly explain their general research findings that might be of interest to the participants. Subsequently, the scientists and participants held a dialogue activity for about an hour at each discussion table. To demonstrate their fields of research, the scientists led their groups in simple games or experiments, such as logic puzzles, easy mock experiments, and constructing concept-representative models.

\section{Interviews}

Among the 27 early-career scientists (including a foreign scientist) participating in the iCeMS Cafes, 19 were interviewed in Japanese. For the interviewees, we did not select a scientist who could not speak Japanese. After having taken part in the iCeMS Cafes, seven scientists had left our institute, so we did not contact them for an interview. All the interviewees were chemical or biological scientists. None of them had previously participated in science cafes as guest speakers, although three had been members of the audience at previous science cafes. Their positions at the time of participating in the iCeMS Cafes were as follows: one lecturer, two assistant professors, seven postdoctoral researchers, six doctoral students, and three master's students.

We used a semi-structured interview format. Each interview lasted for an average of 60 minutes. All interviews were performed by Matsuda, who was not one of the organizers of the iCeMS Cafes and did not participate in any of them. The main lines of questioning in the interviews were as follows: 1) What kind of barriers did the scientists perceive prior to participating in the iCeMS Cafe? 2) What opinions did they have after having participated in the iCeMS Cafe?

With the permission of the interviewees, all interviews were recorded and transcribed. Transcripts were analyzed, using the $\mathrm{KJ}$ method, a technique for analyzing qualitative data derived from Japanese ethnology. ${ }^{28}$ Following this procedure, we conducted five steps in a nonlinear-nonlogical manner, which the method emphasizes so as to free interviewees from a priori assumptions and preconceived notions: 1 ) we coded the transcripts from the interviews and copied the codes to note cards; 2) we shuffled the codes (note cards) and grouped them into "teams"; 3) we assigned titles for all the teams of codes; 4) we arranged the teams into larger "families" and placed titles on the new families; and 5) we devised a spatial patterning of the families into a consistent unifying chart, which had arrows to indicate cause-andeffect relations. 
Two analysis groups, which consisted of two authors each, were involved in the analysis process. First, each group conducted the five steps independently. Second, each group cross-checked the teams, families, and charts. Finally, each group worked together and improved the teams, families, and charts by mutual agreement.

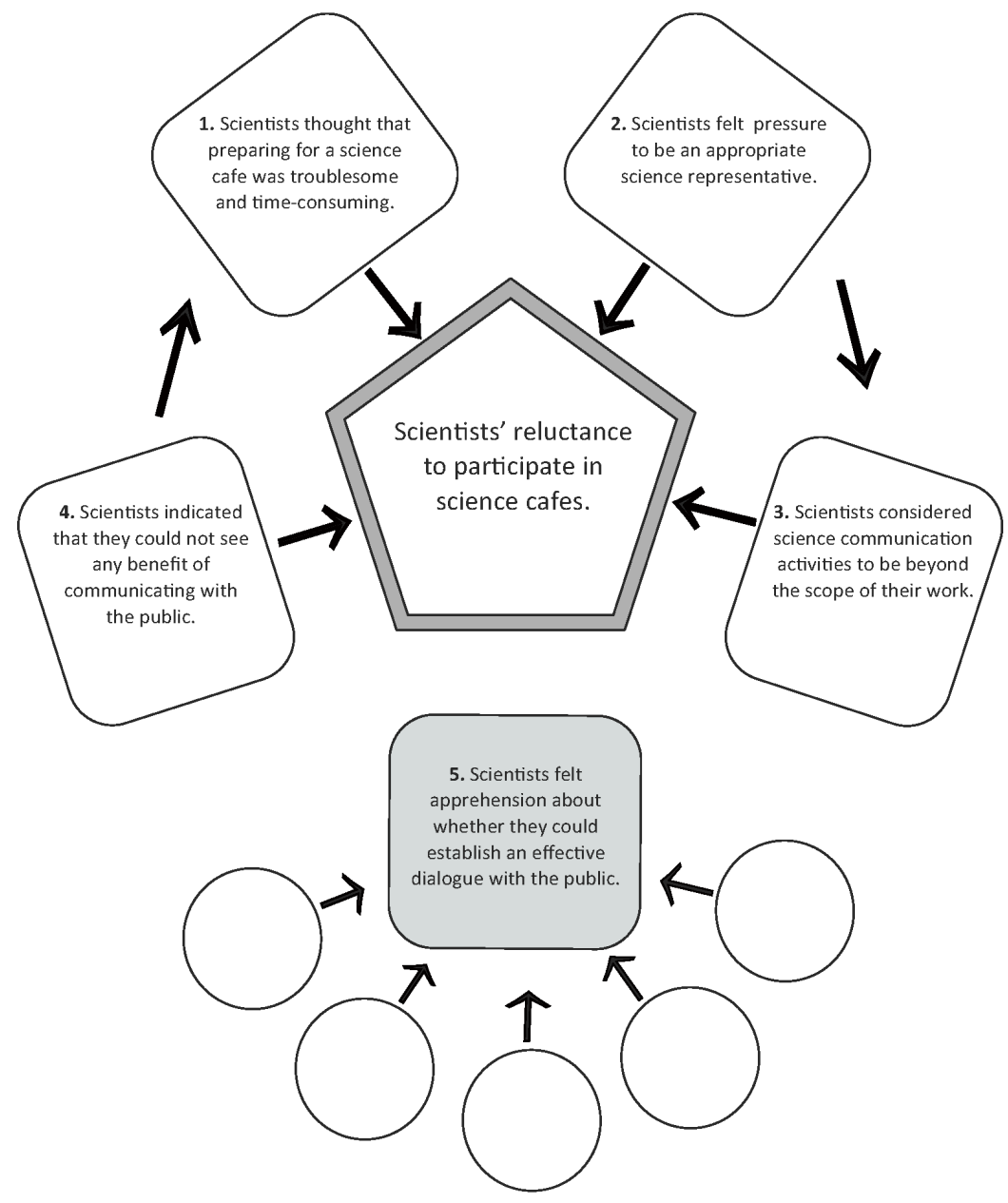

Figure 1. Five primary factors affecting scientists' reluctance to participate in science cafes. The central category of "Scientists' reluctance to participate in science cafes" and the five subcategories (factors), as well as the relationships among them, were extracted from the analyses of interviews using the KJ method. Note that there is no clear connection between the fifth factor, "Scientists felt uneasy about whether they could establish an effective dialogue with the public," and the other categories.

\section{Results}

We deduced five primary factors leading to reluctance to participate in science cafes (Figure 1).

\section{Troublesome and time-consuming}

Many (9 out of 19) scientists indicated that they thought that preparing for a science cafe consumed a lot of their time and effort:

I found it troublesome and time-consuming to prepare a presentation, especially for the lay public (research associate, male, late 30 s at the time of interview). 
And some (four) scientists felt that participating in a science cafe was probably not worth their time:

Participating in the science cafe meant that $\mathrm{I}$ had to spend a great deal of time rearranging my research schedule (assistant professor, male, early $30 \mathrm{~s}$ ).

Moreover, the scientists assigned a low priority to such science communication activities as science cafes. Indeed, some (7) scientists emphasized that they assigned their highest priority to conducting science and research:

I think I should assign a higher priority to writing scientific papers. So I felt like I was doing a bad thing because it didn't involve publishing a paper (research associate, female, early 30s).

In addition, most (16) scientists thought that they needed the assistance of science communicators to help them organize science cafes:

I don't think we can organize science cafes without the people who can properly manage it (research associate, male, late 30s).

When I was at a loss regarding what I should talk about with the participants, the science communicators helped me (assistant professor, male, early 30s).

\section{Pressure to be an appropriate science representative}

Some (six) scientists felt the pressure of creating an effective dialogue with the public as scientific experts:

If we had used complex scientific details in answering a question from the public, that would have caused confusion. Therefore, we often used simplified terms. However, I feared that such simple expressions would cause misunderstandings or misrepresentations ( $\mathrm{PhD}$ student, male, late 20s).

The scientists felt that they should adhere to accurate details of their knowledge because they thought that this was what the public expected of such experts. In addition, some scientists mentioned that they were careful to behave as an appropriate representative of their institute. Therefore, to avoid embarrassment, they often decided that it was better not to directly answer any question that they thought it would be difficult to provide a precise answer for. However, such pressure was in conflict with their desire to deliver accurate scientific knowledge, and this resulted in negative feelings toward the science cafe format.

\section{Outside the scope of their work}

Many (eight) scientists considered that such science communication activities as science cafes went beyond their work responsibilities and commitments. However, some did acknowledge that science communication activities were important, but they insisted that such activities should be restricted to those scientists who are oriented toward science communication or who possess strong presentation skills.

I think that it is important to communicate science with the public, but not all scientists need engage in science communication activities. It is necessary just for certain people to be engaged in this area (lecturer, male, late 30 s).

Others insisted that they had no personal responsibilities or accountabilities regarding the ethical, legal, and social issues that the public is mostly interested in.

Ethical and social issues are out of our range because our research is not directly related to such issues (research associate, male, late 20s).

We found that the scientists' perception of science communication activities as something extraneous to their work seemed to result from the above-mentioned pressure as scientific representatives (Figures 1 and 2). They have little confidence in being able to effectively communicate with the public as experts 
about ethical, legal, and social issues, so they expect it to be done by a presumably more qualified person. In addition, we found that there was some kind of communication barrier in the notion that they assigned a higher priority to science and research work than any other form of science-related work. This means that regardless of the possible benefits of science communication activities, they view such actions as less valuable than directly conducting science and research.

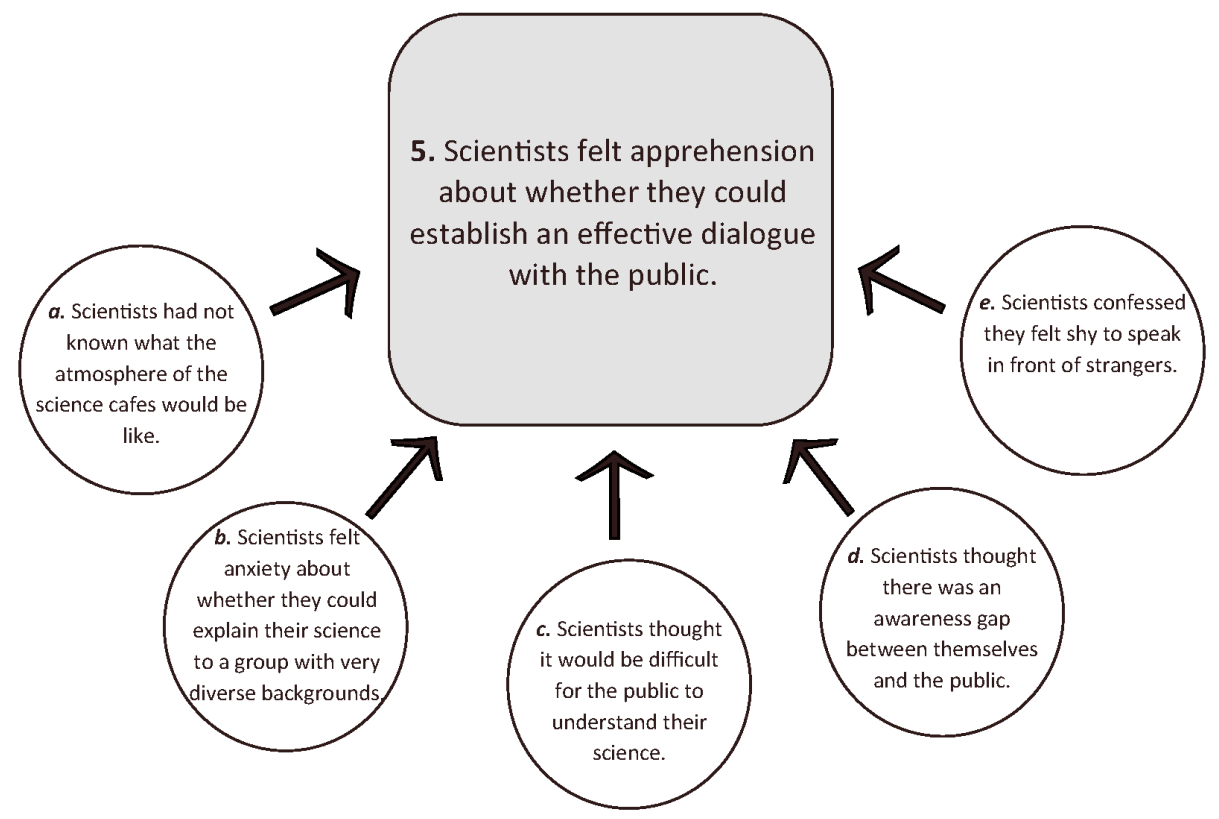

Figure 2. Five reasons why scientists were apprehensive about dialogue with the public. These categories were also extracted from the analyses of interviews using the KJ method.

\section{Could not perceive any benefit}

Many (12) scientists mentioned that they did not see any benefit in communicating the results of their scientific endeavors with the public.

The type of conversation at the science cafe was different from the discussions I hold among colleagues. The talk within the science cafe cannot bring any benefit to my research (lecturer, male, late $30 \mathrm{~s}$ ).

Scientists thought that a dialogue with the public was not meaningful in a scientific context because they could not obtain any ideas from the public that would contribute to their research. Indeed, we noted if scientists could not recognize any benefit, it was also likely to be difficult for the public to understand their research. Moreover, the lack of a perceived benefit reinforced the image of science cafes as being troublesome and time-consuming. As a result, some scientists suggested that the objectives of science cafes should have been explained beforehand:

I could not understand the goal of the science cafe. The concept and the purpose of a science cafe should be made clear to scientists who are participating in one for the first time (lecturer, male, late 30s).

This further indicated that they struggled to develop the motivation to participate in a science cafe. In other responses, we identified two minor opinions. First, one scientist mentioned that the style of science cafes was not efficient because of the very small number of audience participants.

I wanted to share my research with many more people ( $\mathrm{PhD}$ student, male, late $20 \mathrm{~s})$. 
This $\mathrm{PhD}$ student also seemed to regard the science cafe as a place for one-way communication, much like with the mass media, rather than an interactive one.

Second, two scientists thought that science communication activities that targeted junior or senior high school students were necessary:

I should be spreading interest in research among the next generation without regard for any personal benefit (research associate, male, late 30s).

This shows that some scientists would be able to see the benefit of science cafes if they provided the opportunity to communicate with young people who might pursue a career in the sciences.

\section{Apprehension about a dialogue with the public}

Many (12) scientists said that prior to participating in the iCeMS Cafes, they had felt uneasy about whether they could establish an effective dialogue with the public. As illustrated in the analysis shown in Figure 1, they did not explicitly identify feelings of uneasiness about a dialogue with the public as a reason for their reluctance to participate in such science communication activities as science cafes. We found that it was not until after they had participated in a science cafe that the scientists could clearly articulate their unease about a dialogue with the public. Therefore, we identified this factor as the most intrinsic barrier to science communication. In the scientists' comments, we noted five sources of this unease (Figure 2). First, most (16) scientists said that they had felt anxiety about the unknown character of the situation prior to participating in science cafes because they did not know what to expect of such cafes:

I wondered how we should behave at a science cafe $(\mathrm{PhD}$ student, male, late 20s; master's student, female, early 20 s).

I could not imagine who the target audience would be (master's student, female, early 20s; research associate, male, late 20s; research associate, female, early $30 \mathrm{~s}$ ).

Second, many (13) scientists felt anxiety about whether they could properly explain their specific field of science to a group of individuals coming from very different backgrounds:

The participants in the science cafe were quite varied, so I wondered how I should talk with them (research associate, female, early $30 \mathrm{~s}$ ).

Third, many (10) thought it would be difficult for the public to understand their scientific work:

To understand the highlights of our research, some prior knowledge is needed. So even if I made an effort to tell the public about our research, they would not understand it (research associate, male, late 20s).

Fourth, a couple of scientists thought there was an awareness gap between scientists and the public:

Scientists who belong to a university or a research institute cannot guess how they would appear to nonscientists, while nonscientists cannot understand how scientists carry out research (research associate, female, early 30 s).

On the other hand, after experiencing a science cafe, many (12) scientists felt that they were better able to establish a dialogue than they had expected:

The participants in the science cafe asked me some questions that showed an interest in our research, so it was pleasant for me to talk with them (research associate, female, early $30 \mathrm{~s} ; \mathrm{PhD}$ student, male, early $20 \mathrm{~s}$ ).

Furthermore, some scientists regretted that they were not more effective in finding common ground or explaining their research in a more easily understandable fashion:

I should have tried to spend more time listening to the participants and understanding where their interests lay (associate professor, male, early $30 \mathrm{~s}$ ). 
Finally, a few scientists confessed they were embarrassed about speaking in front of strangers:

I am not good at talking to unfamiliar faces. A person who can speak well would know how to behave at a science cafe (research associate, female, early $30 \mathrm{~s}$ ).

\section{Disscussion}

\section{Common barriers to science communication}

We found that scientists thought that preparing for and participating in the science cafe was troublesome and time-consuming (Figure 1.1). This issue is commonly pointed out in research on science communication. ${ }^{1,9,12,13}$ Scientists are already so overburdened that they tend to regard non-research tasks as beyond the scope of their work as well as bothersome. ${ }^{29}$

To address these time constraints, previous studies have argued that a support system for science communication is needed. ${ }^{1,12}$ Indeed, in the present study, many scientists commented that they required the help of science communicators. If science communicators could ease the burden of the scientists' communication responsibility, being able to work with such communicators would be an incentive to participate in science cafes. However, would access to science communicators be sufficient to make scientists more willing to engage in science communication activities? This point has not been clarified in previous research.

\section{Intrinsic barriers to dialogue}

Our results show that it was not until scientists were asked to participate in a dialogue with the public that they spoke of their underlying barriers to such dialogue, as detailed in Figures 1.2 and 2. We revealed how scientists felt pressure from perceived public expectations since they thought they needed to answer questions about wider issues of science outside their own research area (Figure 1.2). Furthermore, in this study, almost all the interviewees were early-career scientists. This may be why they felt particular pressure and unease about presenting themselves to the public as scientific experts. It is possible that early-career scientists have less confidence in communicating with the public about ethical, legal, and social issues. Such scientists may also have little time to think about the societal effects of their research because they have to focus on the demands of their careers. They may also believe that accurate, detailed knowledge about their research is exactly what the public wants. This indicates that early-career scientists need to become more familiar with the aspects of their research that are of interest to the public.

Many scientists worried about whether they could establish an effective dialogue with the participants in a science cafe (Figure 1.5). We identified five reasons for these concerns (Figure 2). First, scientists felt anxiety in the face of an unknown situation, and they felt unease about whether they could explain a complex subject to people from many different backgrounds (Figure 2.a and b). This worry about the unknown environment and the variety of participants would likely be alleviated once the scientists had experienced a science communication activity. Second, the scientists thought it would be difficult for the public to understand their field of science, and they expected there to be an awareness gap between themselves and the public (Figure 2.c and d). Zorn et al. $(2010)^{30}$ reported that public attitudes toward scientists were more positive following a dialogue with scientists, although it was not indicated whether that public fully understood the scientific issues under discussion. Though changes in scientists' attitudes toward the public were not outlined in Zorn et al.'s study, it can be deduced that participation in a science cafe has the potential to bridge the communication gap between scientists and the public. Finally, in the present study, scientists indicated they were embarrassed about speaking in front of strangers (Figure 2.e). This result suggests that scientists' personality traits could affect their apprehension regarding dialogue.

In this study, we succeeded in identifying the more intrinsic barriers to science communication and five reasons causing worry about dialogue with the public; however, there may be additional factors not identified here. The barriers to dialogue may remain even if science communicators help save scientists' time and effort by organizing science communication activities. This suggests that science communicators need to have a better understanding of what scientists are feeling and experiencing 
before, during, and after their dialogue with the public so as to help the scientists reduce the identified barriers. It is possible that once scientists can overcome such negative feelings as anxiety and are able to feel confident about dialogue with the public, they may become highly motivated to participate in science communication activities. Further study on the fundamental barriers to dialogue is needed.

\section{Motivation for dialogue with the public}

It is important not only to reduce the barriers, but also to enhance scientists' motivation regarding dialogue. Shineha et al. $(2009)^{12}$ found that scientists who were most willing to participate in science communication activities tended to enjoy engaging in public dialogue. Furthermore, Jensen et al. $(2008)^{31}$ reported that scientists who actively engage with society are also more academically active. These studies suggest that dialogue with the public need not be tedious or ineffective, but such dialogue may in fact stimulate and promote relevant research. This expected positive-feedback effect on scientists' research could enhance their motivation to engage in science communication activities.

Martín-Sempere et al. $(2008)^{11}$ reported that scientists' strongest motivation to communicate is the desire to increase the public's interest in and enthusiasm for science, their scientific culture, and their awareness and appreciation of science and scientists' contribution. In the present study, we also found that scientists have their own particular goals, motivations, or incentives; for example, some scientists prefer to communicate with young people so as to encourage them to pursue science careers. Thus, scientists can be further encouraged to engage in science communication activities if such activities are relevant to the scientists' own purposes or philosophies.

\section{Benefits of science communication}

Our results show that the scientists did not see any benefits in science communication (Figure 1.3), and they considered it unnecessary additional work (Figure 1.4). These issues have also been pointed out in other studies. 1,9 Thus, we can conclude that scientists are looking for some form of benefit or reward for the effort put into dialogue with the public. Indeed, when asked, some scientists identified possible incentives, including peer recognition or evaluation, personal or career benefits, and money. In a survey by the Royal Society, $20 \%$ of scientist respondents answered that scientists who engage in science communication activities tend to be viewed less highly by their peers. ${ }^{1}$ We attempted to mitigate this effect in the iCeMS Cafes by employing an original format: a principal investigator accompanied by four to seven early-career scientists from the investigator's laboratory participating in the same iCeMS Cafe. This structure of engaging in science communication activities along with their laboratory colleagues could be effective in eliminating the above-mentioned negative associations.

As noted earlier, the Japanese government has recently required scientists who receive more than 30 million yen in public grants to participate in science communication activities, although there is as yet no system in place for evaluating and recording such participation. The importance of evaluation within the scientific community has been accentuated in several reports. ${ }^{1,5,32}$ It is also important to construct a system of rewards for participation that are of value within the scientific community.

\section{Future perspective - designing training programs for scientists}

We conclude that to help scientists remove the identified barriers to dialogue, an appropriate training program is necessary. Some other reports have also pointed out that early-career scientists should be encouraged to participate in science communication activities, ${ }^{1,13}$ so it would be advisable for such scientists to receive training and experience in conducting public dialogue. In fact, several institutions already provide training programs for scientists, such as the American Association for the Advancement of Science, ${ }_{35}^{33}$ the Royal Society, ${ }^{34}$ the Centre for the Public Awareness of Science at Australian National University, $^{35}$ and Econnect Communication Pty Ltd, Brisbane, Australia. ${ }^{8,36}$ These training programs cover strategies to convey key messages clearly to the public or the media. Scientists can have the opportunity to learn how to translate complex science into a widely comprehensible format. These programs emphasize presentation skills, such as giving public lectures and holding press conferences. In 
addition to these components, we would suggest strengthening such programs by adding training sessions to improve scientists' skills related to public dialogue. Such training would include guiding scientists through participation in science communication activities. Based on the influencing factors extracted from the interviews conducted in the present study, we are currently developing a communicationtraining program to help prepare scientists for public dialogue, which we feel should include on-the-job training. Because experience of science communication activities has been clearly shown to be the most powerful predictor of intention to participate in such activities, ${ }^{10}$ scientists may develop a positive impression of science communication by having been guided through that experience. This could serve as a first step for scientists to experience direct communication with the public and would lower the hurdle to participating in more in-depth public deliberations on wider social issues, including policy and ethics of science and technology.

\section{Acknowledgments}

We would like to thank the members of iCeMS, Kyoto University, for their support of the iCeMS Cafes. This study was supported in part by the Research Project on the Deliberation and Cooperation between Citizens and Scientists (DeCoCiS) from Japan's Research Institute for Science and Technology for Society, the Science and Technology Agency to Dr. Hideyuki Hirakawa (and Kazuto Kato as a cooperating researcher), and a Grant-in-Aid for Scientific Research (No. 21830054, to Kei Kano) from the Ministry of Education, Culture, Sports, Science and Technology of Japan.

\section{Notes and references}

1 The Royal Society (2006), Survey of factors affecting science communication by scientists and engineers, The Royal Society, London.

2 A. Zarzeczny et al. (2009), iPS Cells: Mapping the Policy Issues, Cell 139: 1032-1037.

3 E. Kats, F. Soloman, W.P. Mee and R. Lovel (2009), Evolving scientific research governance in Australia: a case study of engaging interested publics in nanotechnology research, Public Understanding of Science 18: 531-545.

4 M. Kurath and P. Gisler (2009), Informing, involving or engaging? Science communication, in the ages of atom-, bio- and nanotechnology, Public Understanding of Science 18: 559-573.

5 J. Illes et al. (2010), Neurotalk: improving the communication of neuroscience research, Nature Reviews Neuroscience 11: 61-69.

6 K. Kato, K. Kano and T. Shirai (2010), Science communication: significance for genome-based personalized medicine-a view from the Asia-Pacific, Current Pharmacogenomics and Personalized Medicine 8: 92-96.

7 M.L. Pace et al., (2010), Communicating with the public: opportunities and rewards for individual ecologists, Frontiers in Ecology and the Environment 8: 292-298.

8 T. Gascoigne and J. Metcalfe (1997), Incentives and impediments to scientists communicating thought the media, Science Communication 18: 265-282.

9 Wellcome Trust/MORI (2000), The role of scientists in public debate, http://www.wellcome.ac.uk/Aboutus/Publications/Reports/Public-engagement/wtd003429.htm.

10 E. Poliakoff and T.L. Webb (2007), What Factors Predict Scientists' Intentions to Participate in Public Engagement of Science Activities?, Science Communication 29: 242-263.

11 M. J. Martín-Sempere, B. Garzón-García and J. Rey-Rocha (2008), Scientists' motivation to communicate science and technology to the public: surveying participants at the Madrid Science Fair, Public Understanding of Science 17: 349-367.

12 R. Shineha, M. Kawakami, K. Kato and A. Hibino (2009), The life science researchers' attitudes toward science communication -motivation, hurdle, and way of promotion, Japanese Journal of Science Communication 6: 17-32.

13 BIOS, London School of Economics and Political Science (2009), Public culture as professional science: final report of the ScoPE project (Scientists on public engagement: from communication to deliberation?), BIOS, London.

14 K.H. Nielsen, C.R. Kjaer and J. Dahlgaard (2007), Scientists and science communication: a Danish survey, Journal of Science Communication 6: 1-12.

15 Ministry of Education, Culture, Sports, Science and Technology (1993), White Paper on Science and Technology 1993.

16 Cabinet council in Japan (1996), First-Stage Basic Plan for Science and Technology (in Japanese), http://www.mext.go.jp/b_menu/shingi/kagaku/kihonkei/honbun.htm.

17 K. Kato, K. Matsuda and H. Morita (2007), Science Communication in Japan: Considering the history and diversity (in Japanese), Protein, Nucleic Acid and Enzyme 52: 1998-2005.

18 Cabinet council in Japan (2001), Second-Stage Basic Plan for Science and Technology (in Japanese), http://www8.cao.go.jp/cstp/kihonkeikaku/honbun.html.

19 T. Kobayashi (2007), The Age of Trans-science: Bridging Science, Technology and Society, NTT Publishing.

20 M. Nakamura (2010), STS in Japan in Light of the Science Café Movement, East Asian Science, Technology and Society: an International Journal 4: 145-151.

21 Council for science and technology policy (2010), Implementation of Public Dialogue on Science and Technology (Basic Policy)

22 D. Dallas (2006), Cafe Scientifique -Déjà Vu, Cell 126: 227-229. 
23 Cafe Scientifique Organisers Conference, Leeds (2007), http://www.muskegoncafescientifique.com/downloads/conferencereport.pdf.

24 J. Mutheu and R. Wanjala (2009), The public, parasites and coffee: the Kenyan Science Café concept, Trends in Parasitology 25: 245.

25 Ministry of Education, Culture, Sports, Science and Technology (2004), White Paper on Science and Technology 2004 http://www.mext.go.jp/english/whitepaper/1302288.htm.

26 K. Matsuda (2008), Science cafe in Japan: a report of the poster exhibition and the workshop about science cafe in Science Agora 2007, Japanese Journal of Science Communication 3: 3-15.

27 M. Nakamura (2008), Science cafe: its scope and challenge, Journal of Science and Technology Studies 5: 31-43.

28 R. Scupin (1997), The KJ method: A technique for analyzing data derived from Japanese ethnology, Human Organization 56: 233-237.

29 Ministry of Education, Culture, Sports, Science and Technology (2004), Survey on R\&D activity in Japan, (In Japanese)

30 T.E. Zorn, J Roper, C. K. Weaver and C. Rigby (2010), Influence in science dialogue: Individual attitude changes as a results of dialogue between laypersons and scientists, Public Understanding of Science (in press).

31 P. Jensen, J-B. Rouquier, P Kreimer and Y. Croissant (2008), Scientists who engage with society perform better academically, Science and Policy 35: 527-541.

32 A.I. Leshner (2007), Outreach Training Needed, Science 315: 161.

33 The American Association for the Advancement of Science, Communicating Science: tools for scientists and engineers, http://communicatingscience.aaas.org/pages/workshops.aspx.

34 The Royal Society, Communication skills and media training courses, http://royalsociety.org/Communication-and-Media-Training/.

35 Centre for the Public Awareness of Science, Science Communication Workshops for Research Scientists, http://cpas.anu.edu.au/workshops/science-communication-workshops-research-scientists.

36 Econnect Communication Pty Ltd., http://www.econnect.com.au/services.htm\#training.

\section{Authors}

Eri Mizumachi, $\mathrm{PhD}$, is a research associate at the Science Communication Group, Institute for Integrated Cell-Material Sciences (iCeMS), Kyoto University, Japan. She also works at Kyoto University Museum as a research assistant. Dr. Mizumachi has coordinated several science cafes and developed educational programs for K-12 students. E-mail: emizumachi@icems.kyoto-u.ac.jp.

Kentaro Matsuda was a research assistant at the Institute for Research in Humanities, Kyoto University, Japan. E-mail: kmatsu00882@gmail.com.

Kei Kano, $\mathrm{PhD}$, is an assistant professor at the Science Communication Group, Institute for Integrated Cell-Material Sciences (iCeMS), Kyoto University, Japan. He has worked widely in science communication for grade school students, adults, and early-career scientists. Dr. Kano is interested in evaluating science communication activities. E-mail: kkano@icems.kyoto-u.ac.jp.

Masahiro Kawakami, $\mathrm{PhD}$, is a researcher in the International Public Communications Office of the Center for iPS Cell Research and Application (CiRA) at Kyoto University, Japan. He is interested in public relations and ethical considerations in stem cell research. Present affiliation: Center for Science Education, Osaka Kyoiku University. E-mail: masakawa@cc.osaka-kyoiku.ac.jp.

Kazuto Kato, $\mathrm{PhD}$, is associate professor of Science Communication and Bioethics at the Institute for Research in Humanities, Graduate School of Biostudies, and Institute for Integrated Cell-Material Sciences (iCeMS), Kyoto University, Japan. His work addresses the ethical and social issues of bioscience including genomics and stem cell research. E-mail: kato@zinbun.kyoto-u.ac.jp.

How to CITE: $\quad$ E. Mizumachi, K. Matsuda, K. Kano, M. Kawakami and K. Kato, Scientists' attitudes toward a dialogue with the public: a study using "science cafes", Jcom 10(04) (2011) $\mathrm{A} 02$. 